\title{
Cognitive behavioral therapy for depression in Japanese Parkinson's disease patients: a pilot study
}

This article was published in the following Dove Press journal:

Neuropsychiatric Disease and Treatment

8 June 2016

Number of times this article has been viewed

\author{
Issei Shinmei ${ }^{1,2}$ \\ Kei Kobayashi ${ }^{3}$ \\ Yuki Oe' \\ Yuriko Takagishi ${ }^{1,4}$ \\ Ayako Kaniel \\ Masaya Ito' \\ Yoshitake Takebayashi ${ }^{1,5}$ \\ Miho Murata ${ }^{3}$ \\ Masaru Horikoshi' \\ Roseanne D Dobkin ${ }^{6}$ \\ 'National Center for Cognitive \\ Behavioral Therapy and Research, \\ National Center of Neurology and \\ Psychiatry, Tokyo, Japan; ${ }^{2}$ Department \\ of Neuropsychiatry, Interdisciplinary \\ Graduate School of Medicine and \\ Engineering, University of Yamanashi, \\ Yamanashi, Japan; ${ }^{3}$ Department of \\ Neurology, National Center of \\ Neurology and Psychiatry, Tokyo, \\ Japan; ${ }^{4}$ Department of Psychology, \\ Surugadai University, Saitama, Japan; \\ ${ }^{5}$ Risk Analysis Research Center, The \\ Institute of Statistical Mathematics, \\ Tokyo, Japan; ${ }^{6}$ Department of \\ Psychiatry, Rutgers-Robert Wood \\ Johnson Medical school, NJ, USA
}

Correspondence: Issei Shinmei National Center for Cognitive Behavior

Therapy and Research, National

Center of Neurology and Psychiatry,

4-I-I Ogawa-Higashi, Kodaira,

Tokyo 187-8553, Japan

Tel +8I 34234627 I

Email issei@ncnp.go.jp
Objectives: This study evaluated the feasibility of cognitive behavioral therapy (CBT) for Japanese Parkinson's disease (PD) patients with depression. To increase cultural acceptability, we developed the CBT program using manga, a type of Japanese comic novel.

Methods: Participants included 19 non-demented PD patients who had depressive symptoms (GRID-Hamilton Rating Scale for Depression score $\geq 8$ ). A CBT program comprising six sessions was individually administered. We evaluated the feasibility and safety of the CBT program in terms of the dropout rate and occurrence of adverse events. The primary outcome was depressive symptom reduction in the GRID-Hamilton Rating Scale for Depression upon completion of CBT. Secondary outcomes included changes in the self-report measures of depression (Beck Depression Inventory-II, Hospital Anxiety and Depression Scale-Depression), anxiety (Hospital Anxiety and Depression Scale-Anxiety, State and Trait Anxiety Inventory, Overall Anxiety Severity and Impairment Scale), functional impairment, and quality of life (Medical Outcomes Study 36-Item Short-Form Health Survey).

Results: Of the 19 participants (mean age $=63.8$ years, standard deviation $[\mathrm{SD}]=9.9$ years; mean Hohen-Yahr score $=1.7, \mathrm{SD}=0.8$ ), one patient ( $5 \%$ ) withdrew. No severe adverse event was observed. The patients reported significant improvements in depression (Hedges' $g=-1.02$, $95 \%$ confidence interval $=-1.62$ to -0.39 ). The effects were maintained over a 3 -month follow-up period. Most of the secondary outcome measurements showed a small-to-moderate but nonsignificant effect size from baseline to post-intervention.

Conclusion: This study provides preliminary evidence that CBT is feasible among Japanese PD patients with depression. Similar approaches may be effective for people with PD from other cultural backgrounds. The results warrant replication in a randomized controlled trial.

Keywords: CBT, anxiety, Japan, dPD, comorbidity

\section{Introduction}

Parkinson's disease (PD) is a neurodegenerative disease associated with disabling nonmotor complications. Depression in individuals with PD is a common neuropsychiatric syndrome, with a prevalence rate of $\sim 50 \%{ }^{1}$ PD depression is highly comorbid with anxiety, closely associated with worsening PD motor function, symptom fluctuations, decreasing quality of life (QOL), and hastening PD progression. ${ }^{2,3}$ Unfortunately, depressive symptoms in PD (dPD) often remain undetected by medical doctors, caregivers, and the patients themselves. ${ }^{4,5}$ The suboptimal recognition of $\mathrm{dPD}$ may be due to the overlapping of somatic features of depressive symptoms (eg, apathy and fatigue) with the motor symptoms of $\mathrm{PD},{ }^{6}$ poor mental health literacy, and the stigma of mental health concerns. ${ }^{7}$

In the treatment of PD depression, antidepressants (selective serotonin reuptake inhibitors and tricyclic antidepressants) are commonly used. ${ }^{8}$ Recent meta-analyses 
have revealed that the effect of antidepressants on dPD was moderate (the pooled effect size was Cohen's $d=0.71$, $95 \%$ confidence interval $[\mathrm{CI}]=-1.33$ to 3.08 ), although not significant, which suggests the need for additional controlled research..$^{9,10}$ Concerns about side effects and polypharmacy are also consistently raised regarding the pharmacological treatment of dPD. ${ }^{11,12}$ Finally, higher anxiety (a common psychiatric comorbidity among dPD) predicts a lower response rate to antidepressants, irrespective of sex or age. ${ }^{13}$ Hence, alternative approaches for depression could be beneficial in PD patients. ${ }^{14}$

Cognitive behavioral therapy (CBT) is an evidence-based psychosocial intervention designed to change dysfunctional thoughts and behavior. A recent meta-analysis of CBT in a non-PD population showed medium-to-large effect sizes for depressive (Hedges' $g=0.45,95 \% \mathrm{CI}=0.25-0.65$ ) and anxiety symptoms (Hedges' $g=0.73,95 \% \mathrm{CI}=0.88-1.65$ ). ${ }^{15}$ Although no large meta-analytic study of dPD has been conducted, one meta-analysis showed that psychosocial intervention including CBT in depressed chronic disease patients versus a control group (standardized care) had a large effect size at posttreatment assessment (standardized mean difference $=-0.84,95 \% \mathrm{CI}=-1.36$ to -0.31$).{ }^{16}$ Two controlled trials of CBT have demonstrated preliminary efficacy in PD. Dobkin et al tested the efficacy of 10 weeks of CBT versus a control group comprising clinical monitoring (depression: Cohen's $d=1.59$, anxiety: Cohen's $d=0.98$ at posttreatment). ${ }^{17}$ Troeung et al also compared 8 weeks of group CBT with a waitlist control group (depression: Cohen's $d=1.12$, anxiety: Cohen's $d=0.89$ at posttreatment)..$^{18}$

However, the feasibility, safety, and effects of CBT for depression in Japanese PD patients with depressive symptoms are unknown. In non-PD populations, it has been shown that Japanese patients are more reluctant to seek professional help as well as social support compared to American patients. ${ }^{19}$ Richards et al also revealed that Japanese patients perceive social distance between "therapists and clients", are skeptical regarding treatment for psychiatric symptoms, and underestimate their ability to enact change in their lives. ${ }^{20}$

Moreover, research in non-PD populations has indicated that depression is both underrecognized and stigmatized in Japanese culture. For example, Shimizu et al found that among Japanese cancer patients with depression in their sample, $>70 \%$ of the patients refused referral for psychiatric treatment. ${ }^{21}$ Japanese patients have also been shown to be more reluctant to discuss depression outside of the family and to have pessimistic views on recovery compared to other cultural groups. ${ }^{22}$ Qualitative data from a large Japanese sample also suggested that depression is viewed as an "excuse" and as a symptom that reflects poorly on people in the public eye. ${ }^{23}$ Although these findings should not be overgeneralized to the entire Japanese population, cultural factors may further impact the underrecognition and suboptimal treatment of dPD in Japan. To popularize CBT among Japanese PD patients, cultural aspects of Japanese reluctance toward clinical help must be considered.

The purpose of the present study was to examine the feasibility of CBT for Japanese PD patients with depression. The CBT protocol previously described by Dobkin ${ }^{17}$ was culturally adapted to meet the needs of the Japanese population. The treatment protocol was shortened and presented with liberal use of cartoon illustrations, a culturally accepted public health information medium in Japan. Specifically, aspects of the Japanese graphic novel style called manga were incorporated into our CBT workbook. Manga can be translated as "comic" in the English language, and manga has captured clinical attention as a useful health care tool. ${ }^{24}$ For example, Imamura et al demonstrated the effectiveness of Internet-based CBT applications with manga on subthreshold depression among Japanese healthy workers, ${ }^{25}$ and it was found to enhance reader's comprehension as well as satisfaction with treatment materials.

We hypothesized that a brief, culturally sensitive CBT program (six sessions) targeting PD depression may be feasible for Japanese PD patients. We also present case examples exploring barriers and facilitators of the treatment process to clarify how Japanese PD patients with depressive symptoms respond to CBT, given the dearth of research in this area.

\section{Methods}

\section{Trial design}

The present clinical trial was an open-label, singlearm study (UMIN Clinical Trials Registry Number: UMIN000016903).

\section{Participants and setting}

This study was approved by the Institutional Review Committee of the National Center of Neurology and Psychiatry (NCNP). All participants were recruited from outpatient clinics and were receiving regular treatment at the NCNP. The potential patients were referred to the National Center for Cognitive Behavior Therapy and Research by neurologists at the NCNP or were self-referred and contacted the center directly. After the referral or contact, the research coordinator gave each participant a full explanation of the 
purposes and procedures related to the study and obtained his/her written consent to participate.

The inclusion criteria were as follows: 1) outpatient receiving regular neurological treatment at the NCNP, 2) primary diagnosis of $P D$ at screening (diagnosed by an NCNP neurologist using the UK Parkinson's Disease Society Brain Bank Clinical Diagnostic Criteria), 3) GRIDHamilton Rating Scale for Depression (GRID-HAMD) score $\geq 8$ (mild depression or more severe) ${ }^{26}$ at screening, and 4) stable dosage of medication, including anti-Parkinson medication and psychotropic medication over the past 6 months. The exclusion criteria were as follows: 1) any alcohol or substance abuse in the past 6 months, 2) psychotic symptoms, 3) diagnosis of dementia, and 4) unstable physical condition.

\section{Intervention}

Our treatment protocol included a maximum of six CBT sessions. No previous study on CBT in Japanese PD patients was available for reference, and we had to base our treatment on the knowledge provided by previous studies reported outside Japan as well as the unique cultural nuances of the Japanese population. As shown in Table 1, previous studies using CBT on PD patients consisted of psycho-education about depression, learning the CBT model, cognitive restructuring, behavioral experiments, and anxiety management approaches. ${ }^{17,18,27,28}$ However, unlike these prior studies, we implemented anxiety management skills (progressive muscle relaxation and breathing exercises) in the earlier phase of our CBT program because of the very high rate of comorbid depression and anxiety in Japan. For example, Yamanishi et al reported that $85 \%$ of depressed Japanese PD patients have comorbid anxiety symptoms. ${ }^{2}$ Addressing anxiety management in the earlier phases of treatment also helped to increase buy-in to the treatment protocol. It was somewhat easier for patients to share concerns about the "stresses" in their lives versus the "depression" they were experiencing.

Previous studies administering CBT to PD patients comprised eight to ten sessions. . $^{17,18,27-29}$ As most Japanese PD patients were unfamiliar with CBT, we considered that a shorter CBT intervention (six sessions) might allow these patients to engage better in CBT. The detailed components of our CBT protocol are shown in Table 2.

A workbook was provided to each participant at no charge. Each session lasted $\sim 60$ minutes. Participants were asked to read a chapter for the next session as homework. At the beginning of each session, a brief assessment of mood was conducted. A review of prior sessions and homework was then conducted. Additionally, participants were asked to attempt the skills learned in the sessions. Feedback related to the homework was written in the workbook.

The program development, including several revisions, was conducted for 2 years prior to the present study. During the fieldwork, several patients commented that it was difficult to read through the workbook, which contained numerous sentences. Therefore, we reduced verbose explanations to the greatest extent possible and adapted culturally accepted illustrations into our CBT materials to increase participants' motivation. As shown in Figure 1, an animated character provided psycho-education on each CBT skill, and all the illustrations were drawn by the lead author (IS). The CBT was provided weekly or biweekly at NCNP's outpatient clinic. We allowed the participants to choose the frequency of their visits based on need and travel time.

Table I Previous study on CBT in PD patients

\begin{tabular}{|c|c|c|}
\hline Study & CBT components & Length \\
\hline Dobkin et $\mathrm{al}^{7}$ & $\begin{array}{l}\text { Behavioral activation, thought monitoring and cognitive restructuring, } \\
\text { relaxation training, worry control, sleep hygiene }\end{array}$ & $\begin{array}{l}\text { Ten individual sessions ( } 60 \text { minutes })+ \\
\text { four caregiver sessions }\end{array}$ \\
\hline Troeung et al $^{18}$ & $\begin{array}{l}\text { Psycho-education, relaxation training, cognitive therapy, problem } \\
\text { solving, and behavioral activation, the role of PD, loss and stress leading } \\
\text { to depression and anxiety, activity scheduling and pacing around the } \\
\text { on-off effect in PD, motor symptoms triggering panic and anxiety, the } \\
\text { fear of falling, and preparing for disease progression }\end{array}$ & Eight group sessions ( 2 hours) \\
\hline Veazey et $\mathrm{al}^{28}$ & $\begin{array}{l}\text { Depression and anxiety education, relaxation training, cognitive therapy, } \\
\text { problem solving, activity scheduling, and sleep management skills }\end{array}$ & Eight telephone sessions \\
\hline Simons et $\mathrm{al}^{27}$ & $\begin{array}{l}\text { Self-monitoring, behavioral health empowerment through pleasant } \\
\text { activities, stress management, anxiety and depression, social } \\
\text { competence, social support }\end{array}$ & Eight group educational programs \\
\hline This study & $\begin{array}{l}\text { Psycho-education on depression and anxiety, anxiety management skill, } \\
\text { cognitive restructuring, behavioral experiment, communication skill, } \\
\text { relapse prevention }\end{array}$ & Six individual sessions \\
\hline
\end{tabular}

Abbreviations: $C B T$, cognitive behavioral therapy; PD, Parkinson's disease. 
Table 2 CBT program components

Session
Session I: CBT model
Session 2: Depression and
anxiety
anxiety

Session 3: Identifying thinking patterns

Session 4: Behavioral experiments skill

Session 6: Review

\section{Components}

Learning the CBT model and understanding the cognitive, behavioral, and biological aspects of $P D$, enhancing realization of how PD affects patients' feelings (frustration, grief, psycho-education on vicious cycle of depression)

Learning how depression and anxiety affect the body and mind and exploring the patients' anxious moments using the CBT model.

Progressive muscle relaxation and breathing exercises are administered Educating on how depression and anxiety might narrow patients' view of the world and complicate the realization of their thinking patterns which are related to their PD symptoms in a daily life setting. Identifying unrealistic or maladaptive thinking patterns and creating other hypotheses against their original thoughts (cognitive restructuring) Based on previous sessions, identify the behavioral aspects influenced by the negative thoughts. The therapist and patient corroborate to create a plan for behavioral experiment to test the negative thoughts Learning communication skills. Reviewing of the understanding of PD symptoms between patients and their family; patients are encouraged to communicate (eg, rehabilitation, pacing of activity)

Evaluating the treatment process and achievement. Relapse prevention for stressful future events

Abbreviations: CBT, cognitive behavioral therapy; PD, Parkinson's disease.

The therapists providing CBT were masters-level psychologists with at least 1 year of clinical experience. The lead author conducted CBT for nine cases (47\%), and other therapists (KK, YO, YT) administered CBT for six, one, and three cases, respectively. To maintain the treatment quality, all therapists were required to receive group supervision once per week. All sessions per participant were supervised on an ongoing basis with audiotaped session recordings. Group supervision was provided by $\mathrm{MH}$ (doctoral-level clinical psychologist). Any questions related to the treatment process, CBT skills, or other medical issues were discussed.

\section{Measures}

The feasibility of CBT in Japan was evaluated via the rate of 1) dropouts and 2) adverse events. The primary outcome

assessed was the reduction in depression as measured using the GRID-HAMD. ${ }^{30}$ Secondary depression outcomes included the Beck Depression Inventory-II (BDI-II) ${ }^{31}$ and the Hospital Anxiety and Depression Scale (HADS). ${ }^{32}$ The Overall Anxiety Severity and Impairment Scale (OASIS), ${ }^{33}$ State and Trait Anxiety Inventory (STAI), and Medical Outcomes Study 36-Item Short-Form Health Survey (SF-36) ${ }^{34}$ were used to evaluate other related outcomes (anxiety and QOL).

All measurement tools were written in Japanese. These assessments were made at the beginning of CBT (Week 0), at the end of treatment ( 6 weeks), and 24 weeks after the end of treatment. Although GRID-HAMD was not conducted blindly, none of the raters had given treatment to the participants whom they interviewed, and a structured interview guide was followed. The raters of GRID-HAMD included the main author (IS) and coauthors (IS, KK, YO, YT).

\section{GRID-Hamilton Rating Scale for Depression}

The Japanese version of the GRID-HAMD is a semi-structured interview based on the administration and scoring of the GRIDHAMD, which was originally developed by Max Hamilton in 1960. The GRID-HAMD (17 items) comprises questions assessing mood, feelings of guilt, suicidal ideation, insomnia, agitation or retardation, anxiety, weight loss, and somatic symptoms. To achieve a detailed description of depressive symptoms, the GRID-HAMD separately evaluates depression frequency, intensity, and severity. The HAMD is the gold-standard measure to use in PD depression treatment trials. ${ }^{35}$ The Japanese version of the GRID-HAMD has excellent reliability (intraclass correlation coefficient $[\mathrm{ICC}]=0.93-0.99) .{ }^{36}$ In the present study, high ICC $(0.93,95 \% \mathrm{CI}=0.58-0.97)$ was also observed (based on ten randomly selected samples) among the four raters (IS, KK, YO, YT). The reliability in the study population was also moderate (Cronbach's alpha $=0.67$ ).

\section{Beck Depression Inventory-II}

The BDI-II, a self-rated questionnaire comprising 21 items on depressive symptoms, is currently used widely in clinical and research fields. Steer et al revised the original version of the BDI-II. ${ }^{31}$ Items are rated from 0 (not at all true) to 3 (very true), and a higher score represents more severe depression. The BDI-II is a commonly employed self-report measure in trials involving PD patients with depressive symptoms. ${ }^{35}$ The reliability in the study population was excellent (Cronbach's alpha $=0.85)$.

\section{Hospital Anxiety and Depression Scale}

The HADS is a 14-item self-rated questionnaire used for evaluating anxiety and depression, developed by Zigmond and Snaith. ${ }^{32}$ Each of the anxiety and depression subscales 


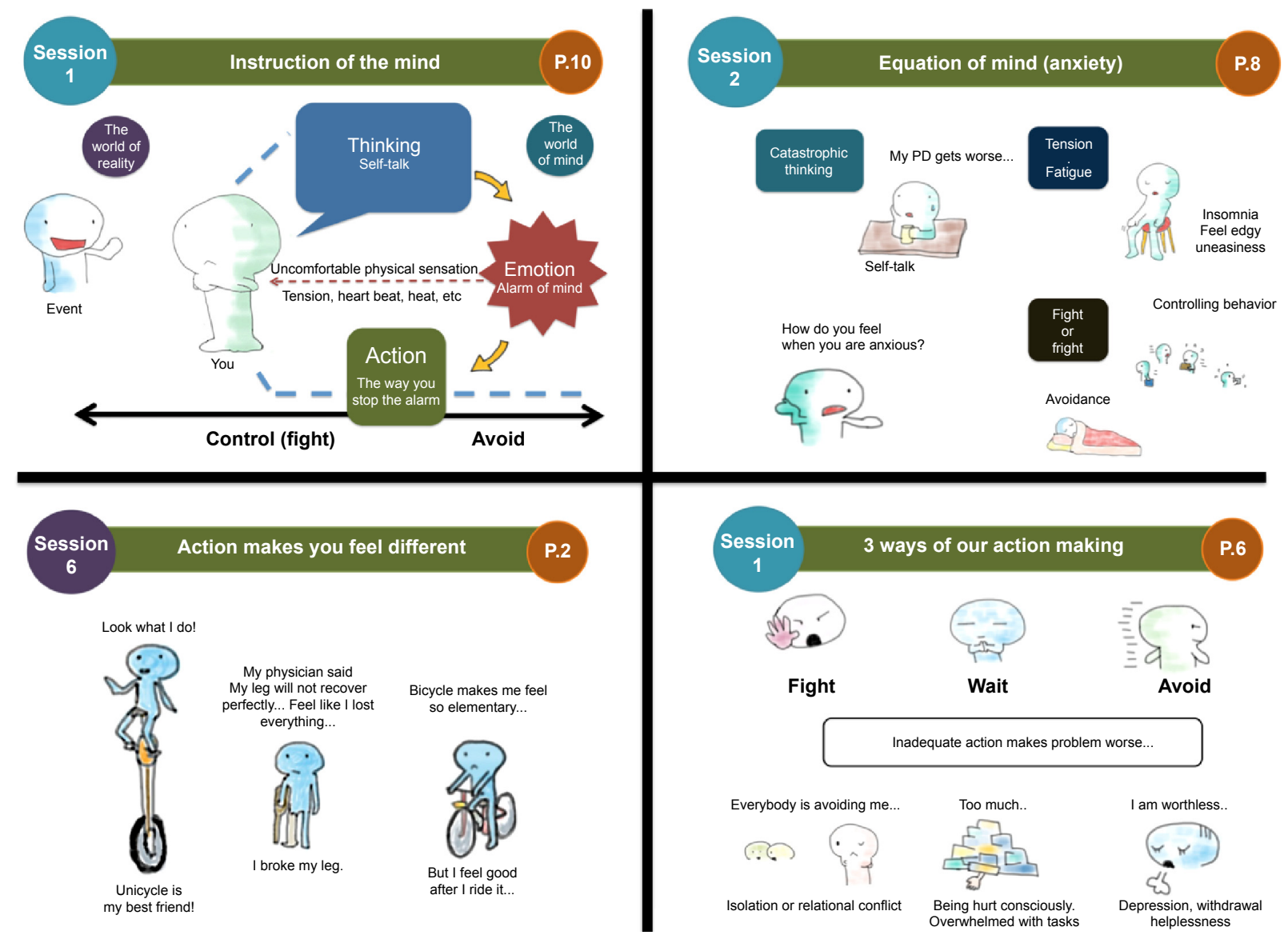

Figure I Components of the patient workbook in this study.

Abbreviation: PD, Parkinson's disease.

includes seven questions, with items scored from 0 (not at all true) to 3 (very true). The HADS was created to evaluate depression (HADS-Depression) and anxiety (HADS-Anxiety) without consideration of physical symptoms, and higher scores indicate more severe anxiety and depression. Marinus et al indicated good reliability (Cronbach's alpha $=0.78-0.86$ ) and moderate convergent validity (Parkinson's Disease Questionnaire, $r=0.72$ ) among 205 Dutch PD patients..$^{37}$ Higashi et al found moderate convergent validity (Zung's self-rating depression scale, $r=0.46-0.57$; and STAI, $r=0.58-0.61) .{ }^{38}$ They also revealed internal consistency when used with Japanese patients with gastrointestinal disorder (Cronbach's alpha $=0.8$ for depression and $0.75-0.76$ for anxiety). ${ }^{39}$ The reliability in the study population was excellent (Cronbach's alpha $=0.86$ for anxiety and 0.85 for depression).

\section{Overall Anxiety Severity and Impairment Scale}

The OASIS $^{33}$ is a five-item simple questionnaire on common symptoms associated with numerous anxiety disorders. Anxiety severity and impairment was calculated with a score from 1 (none) to 4 (constant anxiety, or extreme). A score $\geq 8$ correctly identified $78 \%$ of participants with an anxiety disorder. This measure has moderate convergent validity (STAI, $r=0.68, P<0.00$ ) and excellent internal consistency (Cronbach's alpha $=0.93$ ) in a Japanese psychiatric population. ${ }^{40}$ No previous study for validation of OASIS among PD patients, including Japanese and non-Japanese patients, is available. The reliability in the study population was excellent (Cronbach's alpha $=0.89$ ).

\section{State and Trait Anxiety Inventory}

The STAI is a questionnaire comprising 20 items assessing anxiety. ${ }^{41}$ Although a number of studies using STAI to screen for anxiety in PD exist, no validation study for STAI in the PD population is available. The Japanese version of the STAI has good convergent validity (Manifest Anxiety Scale, $r=0.75$ ) and internal consistency (Cronbach's alpha $=0.83-0.87$ ) among Japanese university students. ${ }^{42,43}$ The reliability in the study population was excellent (Cronbach's alpha $=0.85$ ).

\section{Medical Outcomes Study 36-Item Short-Form Health Survey}

The SF- $36^{34}$ is a self-rated measure of health-related QOL comprising 36 questions within eight subcategories of 
behavioral function and dysfunction (physical functioning, role physical, bodily pain), distress and well-being (vitality, social functioning, role emotional, mental health), and self-evaluations of general health status (general health). Fukuhara et $\mathrm{al}^{34}$ showed moderate-to-high evidence of convergent validity (self-rating depression scale, $r=0.34-0.89$ ) in the Japanese general population. Hagell et al demonstrated good reliability (Cronbach's alpha $=0.78-0.94$ ) in Swedish PD patients. ${ }^{44}$ The reliability in the study population was moderate (Cronbach's alpha $=0.55-0.68$ ).

\section{Sample size}

With the lack of specific data on CBT in Japanese PD patients, we were not able to estimate sample size based on actual data, such as adherence rate and dropout rate. For that reason, we referred to Moore et al's recommendation that CIs should be considered to determine sample size for a pilot study. ${ }^{45}$ Generally, the width of the $\mathrm{CI}^{46,47}$ decreases by increasing the number of participants; however, once an $\mathrm{N}$ of 10 is reached, the difference in CI influenced by the number lessens. By following the suggestion, we determined that our enrollment of 19 participants in the present study was sufficient for a preliminary test of the intervention's effect.

\section{Statistical analysis}

To indicate the standardized mean difference, within-subjects effect size for the mean difference at each time point was computed using Hedges' $g$ with 95\% CIs. We assessed the $95 \%$ CI based on a bias-corrected bootstrap method by generating package bootES in R. ${ }^{48}$ For primary analysis, the change in the primary and secondary outcome variables over time was examined using a linear mixed-effect model (LMM). Based on the intent-to-treat principle, all data from the 19 participants were included in the analysis based on the assumption that missing data were random. ${ }^{49}$ Unconditional growth analyses were undertaken to test a linear individual change trajectory at Level 1 . This trajectory is specified by the individual growth parameter, intercept parameter (nest elevation of the trajectory), and individual slope parameter (rate of change over time). Time-associated changes in data on depression and other related outcome measures were collected at three fixed time points: baseline, posttreatment, and 3-month follow-up. Level 1 individual growth trajectories indicate individual change over the course of study, and Level 2 represents the relationship between individual growth parameters at Level 1 and betweensubject factors. We used restricted maximum likelihood estimation for Levels 1 and 2 because of the small sample size. Additionally, we calculated the proportion of patients meeting criteria for reliable change (outcome measures are significantly greater than that expected by chance given the test-retest reliability of the measure) and clinically significant change (posttreatment scores that better match the distribution of scores in the general population than in a PD sample), based on Jacobson et $\mathrm{al}^{50}$ and Lambert and Bailey. ${ }^{51}$ Normative scores for the measures were obtained from previous studies. ${ }^{37,40,52-54}$ Descriptive analysis and LMM were conducted using SPSS 19.0.1 for Mac (IBM Corporation, Armonk, NY, USA).

\section{Results}

\section{Participant flow and baseline data}

Patients were recruited from April 1, 2012 through August 31, 2014. As shown in Figure 2, we assessed 20 patients. One patient did not meet the inclusion criteria for depression severity. Of the 19 patients included in this study, 18 completed the entire study. On average, it took patients 9.5 weeks

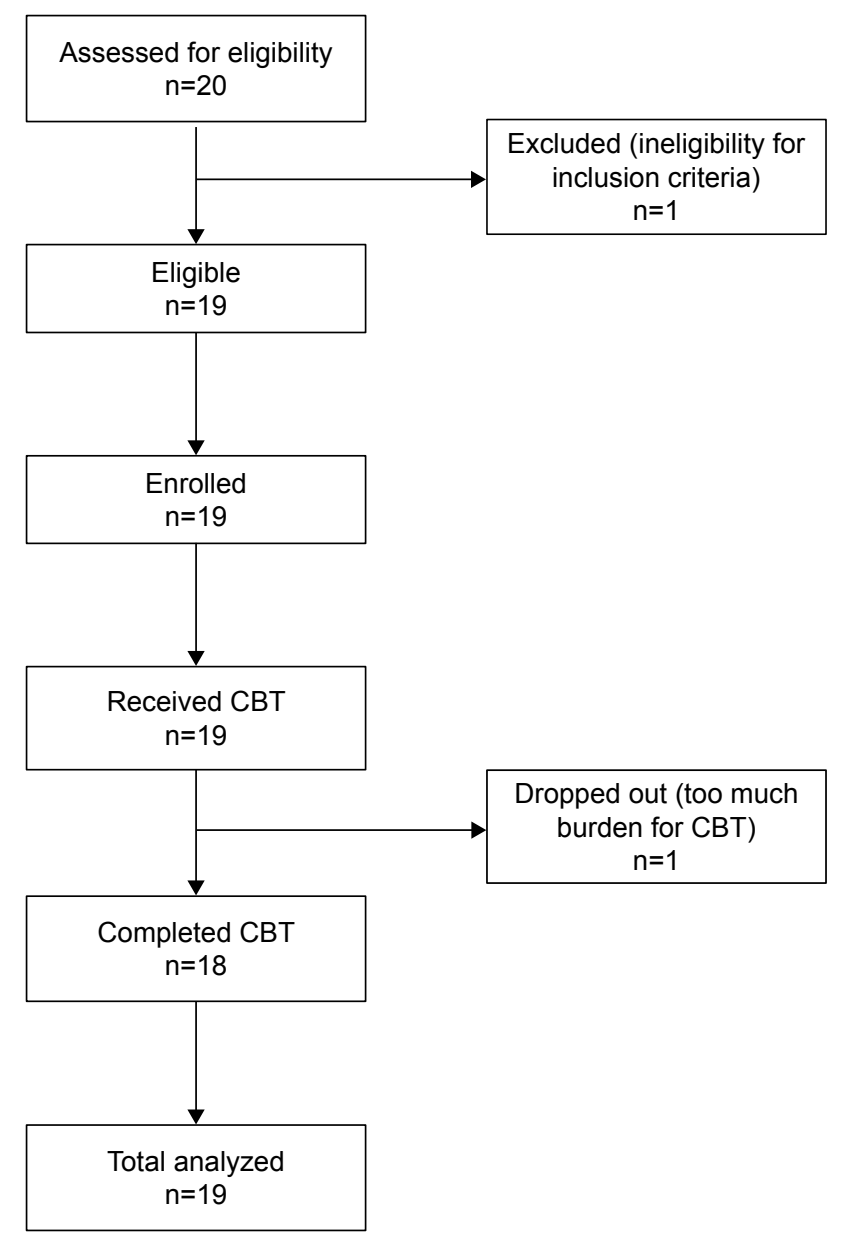

Figure 2 Consort diagram. Abbreviation: $\mathrm{CBT}$, cognitive behavioral therapy. 
Table 3 Participant demographic and clinical characteristics

\begin{tabular}{ll}
\hline Characteristics & \\
\hline Sex (\%) & $7(37)$ \\
Male & $12(63)$ \\
Female & $63.8(9.9)$ \\
Age, mean (SD) (years) & $1.7(0.8)$ \\
Yahr (SD) & $57.4(12)$ \\
Age at onset, mean (SD) (years) & $6.4(3.5)$ \\
Disease duration, mean (SD) (years) & \\
Education (\%) & $5(26)$ \\
High school & $13(68)$ \\
Undergraduate & $1(6)$ \\
Graduate & \\
Marital status (\%) & $2(1 \mathrm{I})$ \\
Single & $16(84)$ \\
Married & $1(5)$ \\
Divorced & \\
Treatment at baseline & $19(100)$ \\
L-Dopa (\%) & $460(268)$ \\
$\quad$ L-Dopa equivalence rate (SD) (mg) & $9(13)$ \\
SSRI (\%) & $4(5.7)$ \\
Equivalent sertraline dose (SD) (mg) & $6(27)$ \\
Minor tranquilizer (\%) & $24(51.7)$ \\
Equivalent diazepam dose (SD) (mg) & $2(7)$ \\
Major tranquilizer (\%) & $0.9(0.7)$ \\
Equivalent chlorpromazine & \\
$\quad$ dose (SD) (mg) & \\
\hline Note Yahr Hohen and &
\end{tabular}

Note: Yahr: Hohen and Yahr staging of Parkinson's disease.

Abbreviations: SD, standard deviation; SSRI, selective serotonin reuptake inhibitor.

(standard deviation $[\mathrm{SD}]=3.2$ ) to complete the six-session CBT protocol. The demographic and clinical characteristics of the study participants are shown in Table 3. The mean age of the 19 participants (seven males and 12 females) was 63.8 years $(\mathrm{SD}=9.9$ years). One patient $(5 \%)$ had obvious wearing off, and the baseline evaluation and posttreatment evaluation for this participant were delivered during the on time. All $(\mathrm{N}=19)$ participants were on anti-Parkinson medications, and they were receiving a stable medication regimen for the duration of the CBT. The L-dopa equivalent dose at each time point was as follows: baseline $=447.4$ $(\mathrm{SD}=283.0)$, posttreatment period $=441.0(\mathrm{SD}=265.4)$, and 3-month follow-up $=435.2$ ( $\mathrm{SD}=254.9)$. Of note, none of our participants had a diagnosis of major depressive disorder at the baseline evaluation.

\section{Dropout and adverse effect}

In the present study, three patients (16\%) reported difficulties engaging in $\mathrm{CBT}$ at the initial session. One patient (5\%) dropped out at the second session. The dropped participant reported that $\mathrm{CBT}$ required tremendous work and that discussing detailed personal issues caused too much pressure.
The other two participants reported feeling anxious about commuting to our hospital because of fear of falling and guilt about increasing their caregivers' burden. We provided all three patients the opportunity to reconsider participating in the study with their caregivers. Two of the patients agreed to continue with the entire session with their caregivers' support (eg, reading the material together, accompaniment during the behavioral experiment). No severe adverse event was observed throughout the trial.

\section{Changes in depression and other related measurements \\ Primary outcome}

Table 4 presents descriptive statistics for the primary and secondary outcome measures throughout the study. Table 5 shows the effect size for each outcome measure (Hedges' $g$ ), and Table 6 shows the parameters of the unconditional growth model for each outcome score.

At baseline, the mean score of the GRID-HAMD was in the moderate range $(14.3, \mathrm{SD}=4.5)$, and it decreased to mild depression $(9.7, \mathrm{SD}=4.3)$ at the posttreatment period. Eight patients (42\%) reported a $50 \%$ decrease in score from the baseline, indicating a clear treatment response (Table 4).

The effect sizes for change in GRID-HAMD scores from baseline to posttreatment (Hedges' $g=-1.02$, 95\% $\mathrm{CI}=-1.62$ to -0.39 ) and baseline to 3 -month follow-up (Hedges' $g=-1.4,95 \% \mathrm{CI}=-1.91$ to -0.78 ) were high (Table 5). The computed width of confidence, which did not include zero, indicated statistical significance of the effect size for the GRID-HAMD change.

For the GRID-HAMD, reliable change and significant change were defined as follows: reliable change score: baseline to posttreatment score $\geq 6$ and baseline to 3 -month follow-up score $\geq 5$; and clinically significant change: baseline to posttreatment score $\geq 8$ and baseline to 3 -month follow-up score $\geq 8$. The proportion of patients meeting the criteria of reliable change was $56 \%$ posttreatment and $50 \%$ at 3-month follow-up. The proportion of patients demonstrating clinically significant changes was $38 \%$ both at posttreatment and at 3-month follow-up.

The unconditional growth model demonstrated significant improvement in the GRID-HAMD at posttreatment $(P<0.01$, estimate $=5.89,95 \% \mathrm{CI}=3.44-8.34)$, while the mean score did not show significant change between posttreatment and the 3-month follow-up ( $P=0.28$, estimate $=1.32,95 \%$ $\mathrm{CI}=-1.13$ to 3.77 ) (Table 6), reflecting a maintenance of treatment gains. 
Table 4 Descriptive statistics

\begin{tabular}{|c|c|c|c|c|c|c|}
\hline \multirow[t]{2}{*}{ Measures } & \multicolumn{2}{|c|}{ Baseline } & \multicolumn{2}{|c|}{ Posttreatment } & \multicolumn{2}{|c|}{ 3-Month FU } \\
\hline & Mean & SD & Mean & SD & Mean & SD \\
\hline \multicolumn{7}{|l|}{ Depression } \\
\hline GRID-HAMD & 14.3 & 4.5 & 9.7 & 4.3 & 8.4 & 3.7 \\
\hline BDI-II & 15.3 & 7.9 & 12.5 & 4.9 & 12.8 & 7.1 \\
\hline HADS-Depression & 7.9 & 4.5 & 5.9 & 4.1 & 6.0 & 3.9 \\
\hline \multicolumn{7}{|l|}{ Anxiety } \\
\hline HADS-Anxiety & 7.1 & 4.1 & 6.1 & 4.0 & 6.3 & 3.7 \\
\hline STAI & 46.4 & 13.0 & 43.6 & 8.0 & 44.5 & 9.9 \\
\hline OASIS & 5.8 & 3.7 & 4.3 & 3.2 & 6.2 & 4.2 \\
\hline \multicolumn{7}{|l|}{ QOL (SF-36) } \\
\hline Physical function & 68.2 & 16.3 & 71.6 & 15.4 & 71.8 & 18.6 \\
\hline Role physical & 58.5 & 27.1 & 64.5 & 23.8 & 68.1 & 26.4 \\
\hline Bodily pain & 52.5 & 25.4 & 57.4 & 23.5 & 52.6 & 23.3 \\
\hline General health perceptions & 36.2 & 14.5 & 36.6 & 14.1 & 38.7 & 21.0 \\
\hline Vitality & 41.1 & 20.6 & 46.3 & 14.2 & 49.0 & 16.8 \\
\hline Social functioning & 64.5 & 24.0 & 65.8 & 26.0 & 72.4 & 23.0 \\
\hline Role emotional & 64.0 & 23.8 & 73.2 & 23.5 & 70.2 & 23.1 \\
\hline Mental health & 61.8 & 17.6 & 68.9 & 12.6 & 66.6 & 16.9 \\
\hline \multicolumn{7}{|l|}{ L-Dopa } \\
\hline L-Dopa equivalence rate (SD) & 447.4 & 283.0 & 441.0 & 265.4 & 435.2 & 254.9 \\
\hline
\end{tabular}

Abbreviations: FU, follow-up; SD, standard deviation; GRID-HAMD, GRID-Hamilton Rating Scale for Depression; BDI-II, Beck Depression Inventory-II; HADS, Hospital Anxiety and Depression Scale; STAI, State and Trait Anxiety Inventory; OASIS, Overall Anxiety Severity and Impairment Scale; QOL, quality of life; SF-36, MOS 36-Item Short-Form Health Survey; MOS, Medical Outcomes Study.

\section{Other related outcomes}

Tables 4-6 also reflect secondary outcome data. The effect sizes for change in self-reported depression measurements were moderate between baseline and posttreatment (BDI-II: Hedges' $g=-0.42,95 \% \mathrm{CI}=-0.97$ to 0.24 ; HADS-Depression: Hedges' $g=-0.44,95 \% \mathrm{CI}=-1.09$ to 0.2$)$ and between baseline and 3-month follow-up (BDI-II: Hedges' $g=-0.32$, 95\% CI $=-0.92$ to 0.37 ; HADS-Depression: Hedges' $g=-0.44,95 \% \mathrm{CI}=-1.1$ to 0.22 ). Regarding anxiety, moderate effect sizes were observed at posttreatment (HADSAnxiety: Hedges' $g=-0.25,95 \% \mathrm{CI}=-0.88$ to 0.40 ; OASIS: Hedges' $g=-0.43,95 \% \mathrm{CI}=-1.06$ to 0.22 ). For QOL (SF-36), two subcategory items showed moderate effect sizes (Role emotional: Hedges' $g=0.38,95 \% \mathrm{CI}=-0.26$ to 1.08 ; Mental health: Hedges' $g=0.45,95 \% \mathrm{CI}=-0.20$ to 1.09 ). In terms of effect size, none of the secondary outcome measures showed statistical significance.

The unconditional growth model indicated that only HADS-Depression $(P<0.05$, estimate $=1.89,95 \%$ $\mathrm{CI}=0.24$ to 3.54 ) and an SF-36 subcategory (Role physical: $P<0.05$, estimate $=-9.59,95 \% \mathrm{CI}=-19.13$ to -0.06$)$ had significant improvement at posttreatment. Both of the outcomes (HADS-Depression: $P=0.95$, estimate $=-0.05,95 \%$ $\mathrm{CI}=-1.70$ to 1.60 ; Role physical: $P=0.45$, estimate $=-3.63$, $95 \% \mathrm{CI}=-13.17$ to 5.90$)$ did not exhibit a significant score change between posttreatment and 3-month follow-up, suggesting maintenance of treatment gains.

\section{Individual therapeutic process}

In addition to the mean score changes represented, the following three cases provide some examples of the therapeutic

Table 5 Outcome measures' ES (Hedge's g)

\begin{tabular}{|c|c|c|c|c|}
\hline & \multicolumn{2}{|c|}{$\begin{array}{l}\text { Baseline to } \\
\text { posttreatment }\end{array}$} & \multicolumn{2}{|c|}{$\begin{array}{l}\text { Baseline to } \\
\text { follow-up }\end{array}$} \\
\hline & $\overline{\text { ES }}$ & $95 \% \mathrm{Cl}$ & ES & $95 \% \mathrm{Cl}$ \\
\hline \multicolumn{5}{|l|}{ Depression } \\
\hline GRID-HAMD & -1.02 & -1.62 to -0.39 & -1.40 & -1.91 to -0.78 \\
\hline BDI-II & -0.42 & -0.97 to 0.24 & -0.32 & -0.92 to 0.37 \\
\hline HADS-Depression & -0.44 & -1.09 to 0.20 & -0.44 & -1.10 to 0.22 \\
\hline \multicolumn{5}{|l|}{ Anxiety } \\
\hline HADS-Anxiety & -0.25 & -0.88 to 0.40 & -0.20 & -0.85 to 0.45 \\
\hline OASIS & -0.43 & -1.06 to 0.22 & 0.10 & -0.53 to 0.75 \\
\hline STAI & -0.25 & -0.85 to 0.42 & -0.16 & -0.78 to 0.52 \\
\hline \multicolumn{5}{|l|}{ QOL (SF-36) } \\
\hline Physical function & 0.21 & -0.44 to 0.86 & 0.21 & -0.46 to 0.83 \\
\hline Role physical & 0.23 & -0.43 to 0.86 & 0.35 & -0.28 to 1.02 \\
\hline Bodily pain & 0.20 & -0.44 to 0.84 & 0.01 & -0.64 to 0.67 \\
\hline $\begin{array}{l}\text { General health } \\
\text { perceptions }\end{array}$ & 0.03 & -0.62 to 0.67 & 0.14 & -0.54 to 0.76 \\
\hline Vitality & 0.29 & -0.36 to 0.95 & 0.41 & -0.26 to 1.08 \\
\hline Social functioning & 0.05 & -0.58 to 0.7 I & 0.33 & -0.29 to 0.96 \\
\hline Role emotional & 0.38 & -0.26 to 1.08 & 0.26 & -0.38 to 0.96 \\
\hline Mental health & 0.45 & -0.20 to 1.09 & 0.27 & -0.37 to 0.90 \\
\hline
\end{tabular}

Abbreviations: $\mathrm{ES}$, effect size; $\mathrm{Cl}$, confidence interval; GRID-HAMD, GRIDHamilton Rating Scale for Depression; BDI-II, Beck Depression Inventory-II; HADS, Hospital Anxiety and Depression Scale; OASIS, Overall Anxiety Severity and Impairment Scale; STAI, State and Trait Anxiety Inventory; QOL, quality of life; SF-36, MOS 36-Item Short-Form Health Survey; MOS, Medical Outcomes Study. 
Table 6 Results for linear mixed model for overall changes with CBT

\begin{tabular}{|c|c|c|c|c|c|c|c|c|c|}
\hline & \multicolumn{3}{|l|}{ Intercept } & \multicolumn{3}{|c|}{ Slope (baseline to posttreatment) } & \multicolumn{3}{|c|}{ Slope (baseline to follow-up) } \\
\hline & Estimate & $P$-value & $95 \% \mathrm{Cl}$ & Estimate & $P$-value & $95 \% \mathrm{Cl}$ & Estimate & $P$-value & $95 \% \mathrm{Cl}$ \\
\hline \multicolumn{10}{|l|}{ Depression } \\
\hline GRID-HAMD & 8.42 & 0.00 & 6.54 to 10.30 & 5.89 & 0.00 & 3.44 to 8.34 & 1.32 & 0.28 & -1.13 to 3.77 \\
\hline BDI-II & 12.84 & 0.00 & 9.80 to 15.88 & 2.47 & 0.10 & -0.50 to 5.44 & -0.32 & 0.83 & -3.29 to 2.65 \\
\hline HADS-Depression & 6.00 & 0.00 & 4.09 to 7.91 & 1.89 & 0.03 & 0.24 to 3.54 & -0.05 & 0.95 & -1.70 to 1.60 \\
\hline \multicolumn{10}{|l|}{ Anxiety } \\
\hline HADS-Anxiety & 6.32 & 0.00 & 4.52 to 8.12 & 0.79 & 0.27 & -0.64 to 2.22 & -0.26 & 0.71 & -1.70 to 1.17 \\
\hline STAI & 44.53 & 0.00 & 39.78 to 49.27 & 1.84 & 0.48 & -3.33 to 7.02 & -0.89 & 0.73 & -6.07 to 4.28 \\
\hline OASIS & 3.37 & 0.00 & I.7I to 5.03 & -0.21 & 0.79 & -1.82 to 1.40 & -0.95 & 0.24 & -2.56 to 0.67 \\
\hline \multicolumn{10}{|l|}{ QOL (SF-36) } \\
\hline Physical functioning & 71.84 & 0.00 & 64.19 to 79.49 & -3.68 & 0.26 & -10.20 to 2.83 & -0.26 & 0.94 & -6.78 to 6.25 \\
\hline Role physical & 68.11 & 0.00 & 56.36 to 79.85 & -9.59 & 0.05 & -19.13 to 0.06 & -3.63 & 0.45 & -13.17 to 5.90 \\
\hline Bodily pain & 52.63 & 0.00 & 41.70 to 63.57 & -0.16 & 0.97 & -9.86 to 9.54 & 4.79 & 0.32 & -4.91 to 14.49 \\
\hline General health perception & 38.74 & 0.00 & 31.17 to 46.30 & -2.53 & 0.56 & -11.16 to 6.11 & -2.11 & 0.62 & -10.74 to 6.53 \\
\hline Vitality & 49.01 & 0.00 & 41.16 to 56.86 & -7.87 & 0.07 & -16.50 to 0.76 & -2.68 & 0.53 & $-\mid \mathrm{I} .3 \mathrm{I}$ to 5.94 \\
\hline Social functioning & 72.37 & 0.00 & 61.36 to 83.37 & -7.89 & 0.17 & -19.38 to 3.59 & -6.61 & 0.25 & -18.09 to 4.88 \\
\hline Role emotional & 70.17 & 0.00 & 59.44 to 80.91 & -6.19 & 0.13 & -14.23 to 1.85 & 3.07 & 0.44 & -4.97 to II.II \\
\hline Mental health & 66.58 & 0.00 & 59.42 to 73.74 & -4.74 & 0.20 & -12.12 to 2.65 & 2.37 & 0.52 & -5.02 to 9.75 \\
\hline
\end{tabular}

Notes: A criterion of significance of $P$-value less than 0.05 .

Abbreviations: CBT, cognitive behavioral therapy; CI, confidence interval; GRID-HAMD, GRID-Hamilton Rating Scale for Depression; BDI-II, Beck Depression InventoryII; HADS, Hospital Anxiety and Depression Scale; STAI, State and Trait Anxiety Inventory; OASIS, Overall Anxiety Severity and Impairment Scale; QOL, quality of life; SF-36, MOS 36-Item Short-Form Health Survey; MOS, Medical Outcomes Study.

techniques and treatment process of this program in Japanese patients. The first two were successful, and the third case showed no improvement.

\section{Case example I}

The first case was a 75-year-old single woman diagnosed with PD 1 year ago. This patient was afraid that someday she would completely lose her physical strength and become totally incapable of living by herself. Although her neurologist told her that her motor symptoms were not as severe as she perceived, she avoided any physical activity as she feared falling and felt ashamed that others would perceive her posture and facial expressions as not socially proper. In this case, anxiety management was a major focus of her treatment. We provided psycho-education, informing her that her excessive worry about falling tightened her muscles and that not only PD symptoms but also anxiety symptoms could cause difficulties in physical activity. Progressive muscle relaxation and breathing exercises were helpful in understanding the controllability of her anxiety symptoms. We also identified cognitions interfering with her daily activities ("falling is unpredictable and could happen anywhere" or "if I meet people, they will automatically assume that I am strange"). We then devised behavioral experiments to examine whether these beliefs were realistic by gradually going outdoors and reconsidering travel to places in which she was less vulnerable to injury. She first attempted going to a coffee shop, which was a place she had been avoiding.
Thereafter, she discovered that most places, except long flights of stairs, were safe enough for her to walk by herself and that the responses of other people were more positive than she expected. Therefore, in this case, identifying exaggerated thoughts and examining those thoughts were essential (GRID-HAMD score decreased from 25 to 18 ).

\section{Case example 2}

The second case was a 70-year-old married woman who exhibited wearing off and was depressed after her close friend passed away. Shortly thereafter, she had an injury to her arm caused by a fall 6 months prior to CBT. Her motto was to always be cheerful and to not express her negative feeling toward others. Before she had depression, she regularly went to the gym for social interaction, as well as rehabilitation to maintain her physical ability. This particular participant was dealing with her depression by keeping it secret. We identified her thoughts ("depression is a sign of weakness" and "my friends will forcefully attempt to explore my situation if I share my concerns with them") and worked to create alternative thoughts by considering several key questions ("If I saw a friend who had the same issue, what would I say to the person? Is there any way to look at the person other than with pity?"). We also created a conversation plan that she would follow with these friends ("If somebody asks how I have been lately, I could simply say, 'I have not been feeling well for a while,' and if these friends attempt to explore in more detail, I also could simply reply, 'I will tell 
you when I feel I am ready"'). Nevertheless, she hesitated to meet with people and even found that sports gear (as she had previously enjoyed athletics and the gym) triggered her fear of meeting with those people. Thus, we constructed a detailed behavioral plan for actually going to meet with friends at the gym and started the process with small steps in which she gradually gathered the sports gear. Afterward, she experimented with the conversation skills acquired during CBT at a sports gym where many of her friends gathered. With this exercise, she learned that her friends' perception of her did not change after her depression, and she felt safe to interact with them even though she had depression. Therefore, the identification of an appropriate behavioral experiment was an important step in improving the patient's QOL. Overall, this patient responded favorably to therapy (GRID-HAMD score decreased from 15 to 2).

\section{Case example 3}

The third case was a married man (64 years old) diagnosed with PD 2 years ago. This patient had an older brother who was in an advanced stage of PD, and being aware of the actual disease progression caused him to be highly anxious. Previously, he ran his own business and often had to communicate with many business-related individuals. After diagnosis, excessive thirstiness and frequent urination, which were probably caused by medication and anxiety, were troublesome. He had concerns about his speech and his ability to fulfill his major role obligations as a father, husband, and provider. He perceived all of his PD symptoms as signs of weakness, and he attempted to hide them from even his own family members. During the CBT, he did homework well and brought his monitoring sheets filled with many sentences. Once he recognized his negative thinking, such as "I am miserable", "I should not reveal my symptoms to others", and "I cannot contribute", he was overwhelmed with anxious feelings and struggled with the need to suppress them. While he was able to successfully use techniques, such as breathing and progressive muscle relaxation, to achieve greater control over his anxiety, he was unable to consistently follow through with the behavioral experiments (eg, share his experiences with friends and family and monitor their reactions) required to directly challenge his negative thinking patterns. From the therapist's perspective, he needed additional sessions to develop the skills needed to more effectively cope with the PD diagnosis and its implications for daily life (baseline GRID-HAMD score was 13 and no score change at posttreatment evaluation).

\section{Discussion}

This study provided preliminary evidence that CBT is feasible among Japanese PD patients who have depression. The dropout rate was low, and no severe adverse events were observed over the course of treatment. The GRID-HAMD, HADS-Depression, and SF-36 (vitality) change scores represented the potential efficacy of CBT among Japanese PD patients with depressive symptoms. However, the statistical analysis showed nonsignificant improvements in most of the secondary measures, including the BDI-II, HADS-Anxiety, STAI, OASIS, and SF-36. Overall, the calculated CIs for efficacy testing were wide.

There was a large within-subjects effect size calculated for the primary outcome (GRID-HAMD; Hedges' $g=-1.02$ ), and our effect on the GRID-HAMD is comparable to that obtained in other studies (eg, within-subject effect size of Hedges' $g=1.58$ with Dobkin et al's CBT, ${ }^{17}$ and Hedges' $g=0.75$ with Troeung et al's $\mathrm{CBT}^{18}$ ). Half of our participants exhibited reliable change at posttreatment, which was interpreted as relative to the effects in prior studies in CBT. However, in the present study, clinically significant change was observed among only $38 \%$ of our participants. Thus, the effect of our CBT on each participant varied, and this may partially explain the wide CI for LMM slope and Hedges' $g$.

Most secondary outcomes did not show statistical significance at any point. The HADS-Depression showed significant change at posttreatment but a smaller effect size (Hedges' $g=-0.42$ ) than that observed in the GRID-HAMD. The reason for the evaluation discrepancy is unclear at this point; however, we assumed that the absence of blind raters and underreporting by the study participants were likely factors at play. Regarding anxiety, none of the selected measurements indicated significant change. The reason for the nonsignificant score change in most of the secondary measurements may be that there was little room for change following treatment, given the low baseline scores. The average baseline scores of the OASIS and HADS-Anxiety were in the nonclinical range (the suggested cut-off point of the OASIS and HADS $=8$ ). ${ }^{39,42}$ Bunevicius et al revealed a cutoff score of 45 for the STAI indicating the presence of anxiety disorder. ${ }^{55}$ The STAI score obtained in the present study was less than the cut-off value, and the severity of anxiety was relatively mild.

Regarding the SF-36 (QOL), nonsignificant changes besides vitality seemed to be influenced by the duration of our CBT as well as many other aspects of the disease characteristics. A recent meta-analysis revealed that positive 
effects of multidimensional interventions (educational program or rehabilitation) among PD patients are inconclusive. ${ }^{56}$ Although depression is most highly correlated with lower QOL in PD, motor symptoms, which CBT does not directly address, also have significant impact on healthrelated QOL. ${ }^{57}$

Additional reasons for insignificant results for many of the measures may be the sample size and duration of treatment. Sample size in the present study was determined by Moore et al's recommendation for a pilot study in that CI should be considered to determine sample size. ${ }^{45}$ According to Diggle et al's method, ${ }^{58}$ the required sample size (power $=0.80$ and alpha $=0.05$ ) is 33 when the effect size is 0.50 . The insignificant results of the secondary outcomes may be due to inadequate sample size and the low power of this study.

Regarding duration of treatment, the addition of a few more sessions may be necessary to help patients generalize the acquired CBT skills in the daily life setting. In our CBT protocol, each theme (eg, cognitive restructuring, behavioral activation) only took place in one session, and time for skill acquisition may have been insufficient. Our biweekly CBT schedule may also have interfered with the skill acquisition process. As the typical interval for the medical examination by neurologists was 2 months and many patients traveled long distances to attend appointments, we allowed biweekly hospital visits for CBT in the present study. A few patients complained about the frequency of hospital visits at the initial session, but they became accustomed to commuting as the sessions were successfully completed. In particular, those patients benefited from family support and encouragement to participate in our study. Since a low dropout rate was observed in these Japanese PD patients with depressive symptoms, reconsideration of intensity and duration may be implemented in future investigations. Of note, Dobkin et $\mathrm{al}^{17}$ included four sessions of family education in their protocol to increase the effect of CBT, and a similar strategy may be used in further research.

Most study participants were in Stage 1 or 2 of PD (the average Yahr score $=1.7, \mathrm{SD}=0.8$ ). All participants were capable of walking into the examination room and writing comments in their workbook with no support. Low-intensity CBT programs, such as ours, for outpatients may be a feasible treatment option, while more advanced PD patients may require special assistance to engage in $\mathrm{CBT}$.

Almost none of our patients had ever heard of CBT before, and they initially tended to be doubtful about CBT and its effectiveness. In order to address these concerns, when obtaining informed consent, our research coordinator showed some components of our CBT workbook and explained the interaction between mental and physical conditions in PD. Most patients were interested in the description and provided consent afterward. In addition, our treatment workbook comprised manga for psycho-education (eg, people generally feel sadness and anger at the loss of health and unknown future caused by disease progression). After reading the material, most PD patients better understood the nature of their depression and anxiety. Thus, buy-in to the CBT program may also contribute to enhanced mental health literacy among PD patients with depressive symptoms in Japan.

\section{Limitations}

The present study should be considered as only preliminary evidence for the efficacy of CBT in Japan, as there are some limitations that require comment. First, this study is limited by the uncontrolled research design with a small sample size and wide-estimated CIs. Second, we did not systematically evaluate therapist treatment fidelity, and the quality of each therapist's CBT competence may not be equivalent. Third, the GRID-HAMD scores may be biased since the raters were not blinded. Fourth, the validity and reliability of the measurements used in the present study were not examined in a Japanese PD population. Fifth, although our CBT program included anxiety management skills, the statistical analyses did not adjust for baseline anxiety. Most of the participants exhibited low baseline scores for anxiety symptoms, and we could not identify how CBT and anxiety symptoms interacted. Sixth, a large number of secondary outcome measures were administered, given the pilot nature of the trial. Thus, Type I error cannot be ruled out as a possible explanation for the few significant findings on secondary self-report measures obtained. Last, we observed a score discrepancy between clinician-rated measures (GRID-HAMD) and self-rated measures (HADS-Depression). This discrepancy may be explained by several factors, including the use of nonblinded raters, participant recall bias on self-report questionnaires, and low mental health literacy in the PD population (which affects reporting on paper-and-pencil questionnaires).

\section{Conclusion}

This is the first study to explore the feasibility and efficacy of CBT for dPD in Japan. Although the present pilot study found CBT to be a feasible and tolerable approach for Japanese PD patients, these results were clearly preliminary and should 
be interpreted with caution. Future CBT research should continue to clarify the effectiveness of CBT in Japanese PD patients, as well as for people with PD from other cultural backgrounds.

\section{Acknowledgments}

This study was funded by the Intramural Research Grant (24-5) for Neurological and Psychiatric Disorders of NCNP. The authors are grateful to Editage as well as Eriko Mizokawa for editorial support.

\section{Disclosure}

No author expects any possible financial gain from the findings presented here. The authors report no conflicts of interest in this work.

\section{References}

1. Reijnders JS, Lousberg R, Leentjens AF. Assessment of depression in Parkinson's disease: the contribution of somatic symptoms to the clinimetric performance of the Hamilton and Montgomery-Asberg rating scales. J Psychosom Res. 2010;68(6):561-565.

2. Yamanishi T, Tachibana H, Oguru M, et al. Anxiety and depression in patients with Parkinson's disease. Intern Med. 2013;52(5): 539-545.

3. Henderson R, Kurlan R, Kersun JM, Como P. Preliminary examination of the comorbidity of anxiety and depression in Parkinson's disease. J Neuropsychiatry Clin Neurosci. 1992;4(3):257-264.

4. Gallagher DA, Schrag A. Psychosis, apathy, depression and anxiety in Parkinson's disease. Neurobiol Dis. 2012;46(3):581-589.

5. Mellilo KD, Houde SC, Sudbury MA. Geropsychiatric and Mental Health Nursing. 2nd ed. Sudbury, MA: Jones \& Bartlett Learning; 2011.

6. Agronin ME, Maletta GJ. Principles and Practice of Geriatric Psychiatry. Philadelphia, PA: Lippincott Williams \& Wilkins; 2011: 473-478.

7. Dobkin RD, Rubino JT, Friedman J, Allen LA, Gara MA, Menza M. Barriers to mental health care utilization in Parkinson's disease. J Geriatr Psychiatry Neurol. 2013;26(2):105-116.

8. Parkinson's Disease: National Clinical Guideline for Diagnosis and Management in Primary and Secondary Care. London: Royal College of Physicians of London; 2006.

9. Skapinakis P, Bakola E, Salanti G, Lewis G, Kyritsis AP, Mavreas V. Efficacy and acceptability of selective serotonin reuptake inhibitors for the treatment of depression in Parkinson's disease: a systematic review and meta-analysis of randomized controlled trials. BMC Neurol. 2010;10:49.

10. Troeung L, Egan SJ, Gasson N. A meta-analysis of randomised placebocontrolled treatment trials for depression and anxiety in Parkinson's disease. PloS ONE. 2013;8(11):e79510.

11. Menza M, Dobkin RD, Marin H, et al. The impact of treatment of depression on quality of life, disability and relapse in patients with Parkinson's disease. Mov Disord. 2009;24(9):1325-1332.

12. Weintraub D, Newberg AB, Cary MS, et al. Striatal dopamine transporter imaging correlates with anxiety and depression symptoms in Parkinson's disease. J Nucl Med. 2005;46(2):227-232.

13. Moonen AJ, Wijers A, Leentjens AF, et al. Severity of depression and anxiety are predictors of response to antidepressant treatment in Parkinson's disease. Parkinsonism Relat Disord. 2014;20(6): 644-646.

14. Zahodne LB, Marsiske M, Bowers D. A latent class analysis of psychological disturbance in Parkinson's disease. Int J Geriatr Psychiatry. 2013;28(10):1054-1060.
15. Hofmann SG, Smits JA. Cognitive-behavioral therapy for adult anxiety disorders: a meta-analysis of randomized placebo-controlled trials. J Clin Psychiatry. 2008;69(4):621-632.

16. Rizzo M, Creed F, Goldberg D, Meader N, Pilling S. A systematic review of non-pharmacological treatments for depression in people with chronic physical health problems. J Psychosom Res. 2011;71(1):18-27.

17. Dobkin RD, Menza M, Allen LA, et al. Cognitive-behavioral therapy for depression in Parkinson's disease: a randomized, controlled trial. Am J Psychiatry. 2011;168(10):1066-1074.

18. Troeung L, Egan SJ, Gasson N. A waitlist-controlled trial of group cognitive behavioural therapy for depression and anxiety in Parkinson's disease. BMC Psychiatry. 2014;14:19.

19. Mojaverian T, Hashimoto T, Kim HS. Cultural differences in professional help seeking: a comparison of Japan and the US. Front Psychol. 2012;3:615.

20. Richards M, Hori H, Sartorius N, Kunugi H. Cross-cultural comparisons of attitudes toward schizophrenia amongst the general population and physicians: a series of web-based surveys in Japan and the United States. Psychiatry Res. 2014;215(2):300-307.

21. Shimizu K, Akechi T, Okamura M, et al. Usefulness of the nurse-assisted screening and psychiatric referral program. Cancer. 2005;103(9): 1949-1956.

22. Nakane Y, Jorm AF, Yoshioka K, Christensen H, Nakane H, Griffiths KM. Public beliefs about causes and risk factors for mental disorders: a comparison of Japan and Australia. BMC Psychiatry. 2005;5:33.

23. Itayama M, Takada E, Tanaka R. Seishinhoken hukushi ni taisuru chiikijyumin no omoi ni kansuru kijutsuteki kenkyu. [Descriptive study on thoughts of local residents toward mentally handicapped people and mental health and welfare]. Hirosaki Reg Repos. 2013;4(1):25-32. Japanese.

24. Kishi Y, Matsumura T, Murishige N, et al. Internet-based survey on medical manga in Japan. Health Commun. 2011;26(7):676-678.

25. Imamura K, Kawakami N, Furukawa TA, et al. Effects of an Internetbased cognitive behavioral therapy (iCBT) program in Manga format on improving subthreshold depressive symptoms among healthy workers: a randomized controlled trial. PLoS One. 2014;9(5):e97167.

26. Zimmerman M, Martinez JH, Young D, Chelminski I, Dalrymple K. Severity classification on the Hamilton Depression Rating Scale. J Affect Disord. 2013;150(2):384-388.

27. Simons G, Thompson SB, Smith Pasqualini MC; for Members of the EduPark consortium. An innovative education programme for people with Parkinson's disease and their carers. Parkinsonism Relat Disord. 2006; 12(8):478-485.

28. Veazey C, Cook KF, Stanley M, Lai EC, Kunik ME. Telephoneadministered cognitive behavioral therapy: a case study of anxiety and depression in Parkinson's disease. J Clin Psychol Med Settings. 2009; 16(3):243-253.

29. Charidimou A, Seamons J, Selai C, Schrag A. The role of cognitivebehavioural therapy for patients with depression in Parkinson's disease. Parkinsons Dis. 2011;2011:737523.

30. Hamilton M. Rating depressive patients. J Clin Psychiatry. 1980;41: $21-24$.

31. Steer RA, Ball R, Ranieri WF, Beck AT. Further evidence for the construct validity of the Beck Depression Inventory-II with psychiatric outpatients. Psychol Rep. 1997;80(2):443-446.

32. Zigmond AS, Snaith RP. The hospital anxiety and depression scale. Acta Psychiatr Scand. 1983;67(6):361-370.

33. Campbell-Sills L, Norman SB, Craske MG, et al. Validation of a brief measure of anxiety-related severity and impairment: the Overall Anxiety Severity and Impairment Scale (OASIS). J Affect Disord. 2009; 112(1-3):92-101.

34. Fukuhara S, Ware JE Jr, Kosinski M, Wada S, Gandek B. Psychometric and clinical tests of validity of the Japanese SF-36 Health Survey. J Clin Epidemiol. 1998;51(11):1045-1053.

35. Schrag A, Dodel R, Spottke A, Bornschein B, Siebert U, Quinn NP. Rate of clinical progression in Parkinson's disease. A prospective study. Mov Disord. 2007;22(7):938-945. 
36. Tabuse H, Kalali A, Azuma H, et al. The new GRID Hamilton Rating Scale for Depression demonstrates excellent inter-rater reliability for inexperienced and experienced raters before and after training. Psychiatry Res. 2007;153(1):61-67.

37. Marinus J, Leentjens AF, Visser M, Stiggelbout AM, van Hilten JJ. Evaluation of the hospital anxiety and depression scale in patients with Parkinson's disease. Clin Neuropharmacol. 2002;25(6):318-324.

38. Higashi A, Yashiro H, Kiyota K, et al. Shokakinaika gairai ni okeru hospital anxiety and depression scale(HAD shakudo) nihongoban no shinraisei to datousei no kentou. [Validation of the hospital anxiety and depression scale in a gastrointestinal clinic]. Jpn J Gastroenterol. 1996;93:884-892. Japanese.

39. Hatta H, Higashi A, Yashiro H, et al. Hospital Anxiety and Depression Scale nihongoban no shinraisei to datousei no kentou: josei wo taishou to shita seiseki. [A validation of the Hospital Anxiety and Depression Scale]. Jpn J Psychosom Med. 1998;38:309-315. Japanese.

40. Ito M, Oe Y, Kato N, et al. Validity and clinical interpretability of Overall Anxiety Severity and Impairment Scale (OASIS). J Affect Disord. 2015;170:217-224.

41. Spielberger CD, Gorsuch RL, Lushene R, Vagg PR, Jacobs GA. Manual for the State-Trait Anxiety Inventory. Consulting Psychologists Press; 1983. Palo Alto, CA, USA.

42. Iwata N, Mishima N, Okabe K, Kobayashi N, Hashiguchi E, Egashira K. Psychometric properties of the State-Trait Anxiety Inventory among Japanese clinical outpatients. J Clin Psychol. 2000;56(6):793-806.

43. Nakazato K, Shimonaka Y. The Japanese State-Trait Anxiety Inventory: age and sex differences. Percept Mot Skills. 1989;69(2):611-617.

44. Hagell P, Tornqvist AL, Hobart J. Testing the SF-36 in Parkinson's disease. Implications for reporting rating scale data. J Neurol. 2008; 255(2):246-254.

45. Moore CG, Carter RE, Nietert PJ, Stewart PW. Recommendations for planning pilot studies in clinical and translational research. Clin Transl Sci. 2011;4(5):332-337.

46. Van Belle G. Statistical Rules of Thumb. John Wiley and Sons, Inc. 2002. Hoboken, NJ, USA.

47. Lee EC, Whitehead AL, Jacques RM, Julious SA. The statistical interpretation of pilot trials: should significance thresholds be reconsidered? BMC Med Res Methodol. 2014;14:41.
48. Little RJA, Rubin DB. Statistical Analysis with Missing Data. New York: Wiley; 2002.

49. Kirby KN, Gerlanc D. BootES: an R package for bootstrap confidence intervals on effect sizes. Behav Res Methods. 2013;45(4):905-927.

50. Jacobson NS, Follette WC, Revenstorf D. Toward a standard definition of clinically significant change. Behav Ther. 1984;17:308-311.

51. Lambert MJ, Bailey RJ. Measures of clinically significant change. In: Cooper H, Camic PM, Long DL, Panter AT, Rindskopf D, Sher KJ, editors. APA Handbook of Research Methods in Psychology, Vol 3: Data Analysis and Research Publication. Washington, DC: American Psychological Association; 2012:147-160.

52. Air T, Weightman MJ, Baune BT. Symptom severity of depressive symptoms impacts on social cognition performance in current but not remitted major depressive disorder. Front Psychol. 2015;6:1118.

53. Matsudaira T, Igarashi H, Kikuchi H, et al. Factor structure of the Hospital Anxiety and Depression Scale in Japanese psychiatric outpatient and student populations. Health Qual Life Outcomes. 2009;7:42.

54. Tokuda Y, Okubo T, Ohde S, et al. Assessing items on the SF-8 Japanese version for health-related quality of life: a psychometric analysis based on the nominal categories model of item response theory. Value Health. 2009;12(4):568-573.

55. Bunevicius A, Staniute M, Brozaitiene J, Pop VJ, Neverauskas J, Bunevicius R. Screening for anxiety disorders in patients with coronary artery disease. Health Qual Life Outcomes. 2013;11:37.

56. Tan SB, Williams AF, Kelly D. Effectiveness of multidisciplinary interventions to improve the quality of life for people with Parkinson's disease: a systematic review. Int J Nurs Stud. 2014;51(1):166-174.

57. Kadastik-Eerme L, Rosenthal M, Paju T, Muldmaa M, Taba P. Health-related quality of life in Parkinson's disease: a cross-sectional study focusing on non-motor symptoms. Health Qual Life Outcomes. 2015;13:83.

58. Diggle PJ, Heagerty PJ, Liang K, Zeger SL. Analysis of Longitudinal Data. Oxford Statistical Science Series. 2nd ed. Oxford: Oxford University Press; 2002.
Neuropsychiatric Disease and Treatment

\section{Publish your work in this journal}

Neuropsychiatric Disease and Treatment is an international, peerreviewed journal of clinical therapeutics and pharmacology focusing on concise rapid reporting of clinical or pre-clinical studies on a range of neuropsychiatric and neurological disorders. This journal is indexed on PubMed Central, the 'PsycINFO' database and CAS,

\section{Dovepress}

and is the official journal of The International Neuropsychiatric Association (INA). The manuscript management system is completely online and includes a very quick and fair peer-review system, which is all easy to use. Visit http://www.dovepress.com/testimonials.php to read real quotes from published authors. 